\title{
Near Surface Phase Transition of Solute Derived Pt Monolayers
}

\author{
Robert E. Rettew $\cdot$ Shuang Cheng $\cdot$ Marc Sauerbrey $\cdot$ \\ Thomas A. Manz • David S. Sholl • Cherno Jaye • \\ Daniel A. Fischer $\cdot$ Faisal M. Alamgir
}

Published online: 18 June 2013

(c) The Author(s) 2013. This article is published with open access at Springerlink.com

\begin{abstract}
As the loadings of precious metals in surfacechemical systems continue to decrease for photo-and electro-catalysts for energy and environmental applications, the study of near-surface electronic and atomic structure in functional materials becomes critically important. Extremely small quantities of active elements, whether grown as clusters or ultrathin films, exhibit changes in catalytic activity that arise from both size effects and electron-transfer effects. These size and transfer effects can be related to increased propensity for oxidation of the metallic deposit, as well as to various changes in electrochemical performance such as durability or required overpotential for a given reaction. This work establishes a minimum threshold for Pt loading beyond which bulk-type electronic behavior may be expected. By iteratively growing atomic monolayers and multilayers using selflimited electrodeposition and studying these films using core-electron spectroscopy (X-ray absorption and X-ray photoelectron spectroscopy), electrochemical methods and
\end{abstract}

Electronic supplementary material The online version of this article (doi:10.1007/s11244-013-0071-9) contains supplementary material, which is available to authorized users.

R. E. Rettew · S. Cheng · M. Sauerbrey · F. M. Alamgir $(\bowtie)$

School of Materials Science and Engineering, Georgia Institute of Technology, Atlanta, GA, USA

e-mail: faisal.alamgir@mse.gatech.edu

T. A. Manz · D. S. Sholl

School of Chemical and Biomolecular Engineering, Georgia Institute of Technology, Atlanta, GA, USA

C. Jaye $\cdot$ D. A. Fischer

National Institute of Standards and Technology, Gaithersburg, MD, USA
DFT-based computations the fundamental interactions that govern oxidation state and electron transfer near the surface of a Pt-Au bimetallic system have been explored. It has been shown that the Pt-Au system exhibits increased tendency for the Pt layer to remain cationic below a minimum threshold film thickness of two monolayers. At monodispersed levels of submonolayer coverage $\mathrm{Pt}$ exhibits deviated electronic structure, reactivity, and metal stability compared to films in excess of this minimum threshold thickness. At three monolayers Pt is thick enough to avoid the preference for cationicity and the resulting higher rates of metal dissolution, but thin enough to benefit from electron transfers from $\mathrm{Au}$ that assist in lowering the overpotentials for $\mathrm{CO}$ oxidation. This study shows the efficacy of a concerted method for the investigation of near-surface phenomena in multicomponent systems. By combining electrochemical and vacuum studies of solutederived samples with advanced computational techniques, a multifaceted understanding of these architectures has been achieved.

Keywords Platinum - DFT - XPS - XANES - EXAFS . Fuel cell $\cdot$ Monolayer

\section{Introduction}

The near-surface compositional architecture in photo, electro, and traditional heterogeneous catalyst systems fundamentally shapes their properties. Design of nearsurface composition often employs synthesis through solution chemistry where metal ions are reduced onto a support surface. Nominally, it is understood that as metal layers build, the reduction reaction is complete and the properties of the adlayer metal may be described by that of 
neutrally charged atoms. There is also an expected threshold lengthscale over which the support material affects the surface chemistry through electron transfers. However, for very low adlayer thicknesses of deposited species, it is not clear whether the deposit prefers to remain partially charged through effects of either low dimensionality or charge transfer with the substrate. At the theoretical lowest-loading limit, where single atoms are deposited in monodispersed geometries and adatom-adatom distances are large enough that lateral interactions can be neglected, the tendency of an adatom to oxidize or reduce may be quite different than that of the bulk material.

This preference itself may transition from deposits of sub-monolayers (which offer the possibility of monodispersed growth modes) to a few monolayers and beyond. Past a threshold adlayer thickness, the effect of electrons transferred between the support material and the surface atoms plays no detectible role on the surface chemistry of the deposited metal. There is, therefore, an expected minimum thickness below which adlayer deposits may prefer to remain cationic and a maximum thickness beyond which the support plays no role on the surface chemistry. These two critical dimensions have implications for issues in catalysis such as: minimum amount of precious metal loading, precious metal durability, catalytic activity, overpotential for electrocatalytic reactions, electron-hole recombination rates, and plasmonic coupling effects (relevant in photocatalysis).

$\mathrm{Pt}$ is of interest due to its properties as a catalyst for a wide range of reactions. $\mathrm{Pt}$, whether in alloy or adlayer form, shows desirable properties that may be tuned by the presence of a neighboring metal at the nano or atomic scale [1]. The knowledge of these changes and the length scale over which they occur is necessary to mitigate negative effects and to discover beneficial properties arising from the proximity of dissimilar metal atoms. Near-surface changes in behavior of Pt have recently been observed but until now no systematic, layer-by-layer study of solutederived $\mathrm{Pt}$ and its near-surface phase transitions has been conducted $[2,3]$.

Recent work has addressed the treatment of substrateoverlayer systems by theoretical methods [4, 5]. These studies indicated that novel phenomena may be observed for such ultrathin overlayers, but specialized techniques are required to fabricate these surfaces. A review paper by Chen et al. [6] summarizes the body of ultra-high-vacuum and density functional work on this type of overlayersupport system. In contrast to these works, this study uses an electrochemical technique called surface limited redox replacement (SLRR) to create $\mathrm{Pt}-\mathrm{Au}$ adlayer-substrate samples to study these properties. SLRR allows for electrodeposition processes with atomic monolayer-level and submonolayer-level precision. This permits the use of small amounts of elements such as Pt and allows the discovery and design of surface electronic structure-dependent properties. This technique, which was employed by Adzic, Brankovic, and Sasaki for the growth of Pt monolayer catalysts [7], was used by Weaver [8] to grow several atomic multilayers of $\mathrm{Pt}$ and has been expanded upon and studied by several authors $[9,10]$.

This work systematically relates the electronic and atomic structure of $\mathrm{Pt}$ films on Au to chemical reactions that are important to applications in fuel cells, water splitting, and carbon monoxide conversion. By studying a series of Pt films, it is possible to both approach the lowloading monodispersion limit (for submonolayer deposits) and assign a length scale to substrate-adlayer electronic exchange effects (for multilayer deposits). In the progressive growth of adlayer $\mathrm{Pt}$ on $\mathrm{Au}$, its electronic/atomic structure (measured by core electron spectroscopy) reveal near-surface phase transformation from a cationic to a more metallic nature of Pt. Unique features of this transformation are corroborated by DFT based calculations on representative $\mathrm{Pt} / \mathrm{Au}$ adlayer/support architectures.

\section{Experimental}

Electrodes were either Au-coated glass slides or polycrystalline Au discs polished to $50 \mathrm{~nm}$ using Buehler alumina suspensions mixed with $\mathrm{H}_{2} \mathrm{O}_{2}$, then immediately cleaned ultrasonically in DI water and isopropyl alcohol. Solutions were de-aerated using dry nitrogen before being used. The precursor solution was $10 \mathrm{mM} \mathrm{CuSO}_{4}+50 \mathrm{mM}$ $\mathrm{H}_{2} \mathrm{SO}_{4}$. The Pt replacement bath used was $1 \mathrm{mM} \mathrm{H}_{2} \mathrm{PtCl}_{6}$ in DI water (18.2 M $\Omega)$. Voltammetry was conducted using a Pine WaveNow potentiostat and a Solartron 1287 potentiostat with homemade $\mathrm{Ag} / \mathrm{AgCl}$ reference electrodes calibrated to a commercially available reference. All samples were rinsed in deaerated $1 \mathrm{M} \mathrm{H}_{2} \mathrm{SO}_{4}$ and dried in nitrogen after fabrication. Gasses used were of $99.99 \%$ purity $\left(\mathrm{N}_{2}\right)$ or $99.9 \%$ purity $(\mathrm{CO})$. X-ray photoelectron spectroscopy (XPS) was conducted using a high resolution Thermo K-Alpha spectrometer. X-ray absorption spectroscopy (XAS) was conducted at beamlines X23A2 and $\mathrm{X} 18 \mathrm{~B}$ at the National Synchrotron Light Source, Brookhaven National Laboratory. XAS analysis was performed using Athena, and XPS fitting was performed using XPSPeak software.

Samples were fabricated by plating of controlled submonolayer $\mathrm{Cu}$ precursor deposits in one cell, followed by emersion of the sample from the $\mathrm{Cu}$ bath and immersion in a $1 \mathrm{mM} \mathrm{H}_{2} \mathrm{PtCl}_{6}$ replacement bath for $10 \mathrm{~min}$ at open circuit. Coverage of Pt deposits was controlled by use of $\mathrm{Cu}$ underpotential deposition (UPD) and manipulation of the emersion potential. $\mathrm{Cu}$ UPD is a well-studied process 
and is known to proceed in two waves, with the first wave depositing 2/3 of a monolayer and the second wave completing the full monolayer coverage [11]. By removing samples from the $\mathrm{Cu}$ bath at a potential between these two deposition waves, we were able to limit the $\mathrm{Cu}$ deposit to $\sim 0.6 \mathrm{ML}$ per iteration. Due to the $2: 1 \mathrm{Pt}: \mathrm{Cu}$ replacement ratio, it is possible to determine the nominal Pt loading of a given sample in terms of monolayerequivalent (ML-EQ) charge, with one iteration of the growth cycle corresponding to 0.3ML-EQ of Pt. By repeating multiple growth iterations, increasing coverages (0.6ML-EQ, etc.) were achieved. The use of $\mathrm{Pt}^{4+}$ as replacing cation allowed for higher resolution of $\mathrm{Pt}$ growth to be achieved as compared to $\mathrm{Pt}^{2+}$, which would have grown twice as much $\mathrm{Pt}$ per iteration. Accelerated aging tests were conducted in $1 \mathrm{M} \mathrm{H}_{2} \mathrm{SO}_{4}+0.5 \mathrm{M}$ Methanol using carbon fiber paper as-purchased. Carbon K-edge XANES was conducted after electrochemical adsorption of $\mathrm{CO}$ from a CO-saturated $\mathrm{H}_{2} \mathrm{SO}_{4}$ bath followed by transfer in $\mathrm{N}_{2}$ atmosphere to beamline U7A at the National Synchrotron Light Source.

Plane-wave density functional theory calculations were performed using the Vienna Ab Initio Simulation Package (VASP) $[12,13]$. The all-electron frozen core projector augmented wave (PAW) method [14] was used with a $400 \mathrm{eV}$ plane-wave cutoff. Calculations were performed using a $3 \times 3 \times 1$ Monkhorst-Pack k-point grid and the gaussian smearing method with a $0.1 \mathrm{eV}$ smearing width. The PW91 exchange-correlation functional with the correlation interpolation of Vosko et al. [15, 16] was used. Geometries were optimized to give forces of less than $0.03 \mathrm{eV} / \AA$ on all atoms. The supercells contained five $\mathrm{Au}$ layers (with nine $\mathrm{Au}$ atoms per layer) plus zero to three $\mathrm{Pt}$ adlayers with $\sim 10 \AA$ vacuum spacing between slabs. DDEC charges were computed using the DDEC method of Manz and Sholl [17]. The DFT optimized lattice constant for bulk Au (4.17 $\AA$ ) was used. A more complete explanation of DFT measurements is available in the supplemental material.

\section{Discussion 1: Film Characterization}

Film characterization has revealed two significant phenomena. The first is the presence of cationic surface Pt for film thicknesses of less than 2ML-EQ, while the second arises from electron transfer with the Au substrate and the metallic component of the Pt film. In this first section we address the evidence for the existence of the cationic species as seen in XPS and XAS, and the implications of this finding on the nominal SLRR growth mechanism. The two electrochemical equations that are traditionally used to describe SLRR growth are given in Eqs. 1 and 2.
$\mathrm{Cu}^{2+}+2 e^{-} \rightarrow \mathrm{Cu}$

$2 \mathrm{Cu}+\mathrm{Pt}^{4+} \rightarrow \mathrm{Pt}+2 \mathrm{Cu}^{2+}$

The amount of noble metal grown is restricted by the amount of precursor on the surface (which is limited by UPD). This can then be repeated iteratively to grow multilayer deposits. This study shows that the above reaction pathway is only approximately accurate for very small iteration numbers in specific bath chemistries, and that the Pt film grown is partially cationic. A recent study by Brankovic et al. [18] focused on the oxidation state of the dissolved anionic species created during dissolution of the $\mathrm{Cu}$ adlayer and reported that the $\mathrm{Pt}-\mathrm{Cu}$ replacement reaction does not always behave according to the traditional model. It is clear that the exact reaction mechanism for the SLRR process is not yet fully understood. The cationic nature of the film decreases with increasing monolayer thickness as portrayed schematically in Fig. 1.

Since Pt nanostructures are commonly grown from solutions containing $\mathrm{Pt}^{4+}$ ions by spontaneous deposition, potentiostatic or pulsed electrodeposition and precipitation, there is a presumed reduction of $\mathrm{Pt}^{4+}$ ions to their metallic state in all of these synthesis routes as Pt atomic layers stack and grow [19]. In principle, SLRR growth is a window through which one can study the characteristics of the initial stages of deposition common to all of these techniques.

\subsection{Evidence for Cationic Content of Film}

Figure 2 contains the XPS spectra of several different coverages of Pt grown on Au using the SLRR method. Two

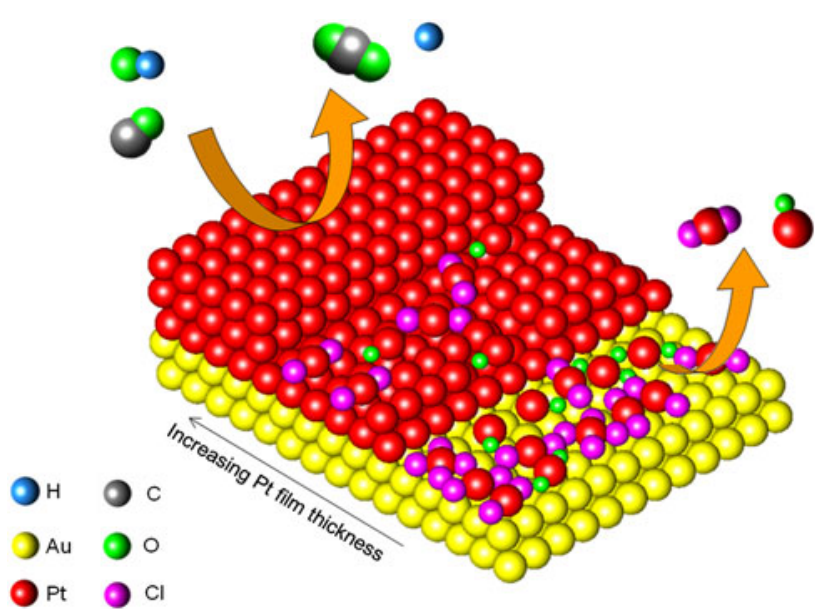

Fig. 1 Schematic of the Pt-Au surface for three different thicknesses of Pt, indicating the prevalence of cation-anion complexes for thinner $\mathrm{Pt}$ deposits. Reaction arrows (orange) indicate $\mathrm{CO}$ electro-oxidation (left) and film dissolution (right) 
reference curves provide spectra for $\mathrm{Pt}^{4+}\left(\mathrm{PtO}_{2}, \mathrm{H}_{2} \mathrm{PtCl}_{6}\right)$ and one reference curve for metallic Pt. Based on these spectra we determined the coexistence of various oxidation states of Pt in the SLRR-grown film. For the spontaneously grown and 0.3ML-EQ curves, multiple features are apparent, indicating the presence of $\mathrm{Pt}^{4+}\left(4 \mathrm{f}_{7 / 2}\right.$ at $\left.74.4 \mathrm{eV}\right)$ and metallic $\mathrm{Pt}\left(4 \mathrm{f}_{7 / 2}\right.$ at $\left.71.1 \mathrm{eV}\right)$, as well as a new feature near $72.2 \mathrm{eV}$ that corresponds to the $4 \mathrm{f}_{7 / 2}$ peak of $\mathrm{Pt}^{2+}$ species [20]. With increasing Pt coverage, the amount of $\mathrm{Pt}^{4+}$ and $\mathrm{Pt}^{2+}$ decreases and the amount of metallic $\mathrm{Pt}$ increases. After six iterations, there is a very small amount of $\mathrm{Pt}^{2+}$ present and the majority of signal arises from the metallic Pt. It is important to remember that reduction of cationic $\mathrm{Pt}$ during subsequent $\mathrm{Cu}$ deposition is inherent to SLRR. In this way it is possible for the $\mathrm{Pt}^{4+}$ created in the first iteration to be eliminated during the second iteration's $\mathrm{Cu}$ growth step. Figure $2 \mathrm{~b}$ graphically portrays the results of DFT calculations of surface atomic charge conducted for three representative architectures similar to those grown by SLRR. For modeled architectures of Pt overlayer coverage less than $0.5 \mathrm{ML}-\mathrm{EQ}$, we see a slight increase in propensity for Pt adatoms to remain positively charged. This structureinduced inclination towards a slightly positive charge induces the formal oxidation state change observed in XPS. DFT analysis of the $1 \mathrm{ML}$ case indicates electron transfer from gold to the first fully formed Pt layer, followed by neutral charge for the second layer. This trend is also apparent in XPS (Fig. 2c) and will be discussed in Sect. "Evidence for Electron Transfer in Pt Film".

Further evidence for the presence of cationic Pt species is presented in the XAS analysis (Fig. 3). For both the 1 ML-EQ and 2ML-EQ samples, significant deviation in first-neighbor location peaks compared to metal foil are seen. This is consistent with previous reports of bulk materials [21, 22]. By examining Figs. 3a, b, we compare the thin films with reference spectra of $\mathrm{Pt}-\mathrm{O}, \mathrm{Pt}-\mathrm{Cl}$, and metallic Pt-Pt bonds. It is clear that the as-prepared 1MLEQ SLRR sample exhibits a shorter first neighbor distance. This indicates a cationic surface species with both $\mathrm{Cl}$ and $\mathrm{O}$ present. Meanwhile, the as-prepared 2ML-EQ sample exhibits a first-neighbor distance close to that of $\mathrm{Pt}-\mathrm{Cl}$ bonding, although the peak is broad enough to permit $\mathrm{Pt}-\mathrm{O}$ bonding as well. After an electrochemical reduction step, the 2ML-EQ Pt deposit has converted to Pt similar to that in the bulk foil, while the 1ML-EQ deposit still shows a significant shift to lower interatomic distances, indicating residual cationicity. The increase in peak intensity from the 2ML-EQ as-prepared sample to the 2ML-EQ reduced sample indicates an increase in ordering of the deposit. It is also apparent that the reduced 1ML-EQ deposit is still dominated by the residual oxide feature, indicating that no such ordering of the metallic phase has occurred.

\subsection{Evidence for Electron Transfer in Pt Film}

The trend in zero-valent $\mathrm{Pt} 4 \mathrm{f}_{7 / 2}$ binding energy location mentioned is apparent as a shift in peak location of the metallic component of the XPS spectrum (Fig. 2c). This negative shift is largest for small iteration numbers $(-0.4 \mathrm{eV})$. After more iterations, there is still a deviation of $-0.2 \mathrm{eV}$ compared to bulk-type photoemission spectra. It has been shown that these types of trends can be correlated with trends in the d-band center [23]. When considering these types of effects, we must consider both electron transfer from the Au substrate and lateral interactions with coexisting cationic $\mathrm{Pt}$ on the surface.

To discern the role of the Au substrate in this process, we performed one iteration of SLRR growth on a Pt substrate. This shifted the photoemission line to lower binding energies, but not to the extent seen on the Au substrate. These results indicate that a portion of the shift is dependent on the substrate, and another portion is dependent on the deposit itself. To track the influence of the cationic Pt in the film on the binding energy of the metallic Pt in the film, we measured the binding energy of the metallic component after electrochemical reduction at $0 \mathrm{~V}$ in $\mathrm{H}_{2} \mathrm{SO}_{4}$ (denoted as ML-EQ reduced). The negative shift in binding energy decreased in magnitude after reduction. This correlates with a decrease in the amount of cationic Pt in the film. Since the negative shift was not eliminated by either the use of a Pt substrate or the electrochemical reduction step, we conclude that these shifts are due to: (1) The identity of the substrate, (2) the degree of cationicity in the film, and (3) the thickness of the film. As shown in supplementary Table 1, DFT calculations confirm a small amount of surface anionicity $(\sim-0.02$ per $\mathrm{Pt}$ atom) remains as the number of Pt layers increases.

The analysis of the intensity at $4 \mathrm{eV}$ in the XANES spectra (Fig. 3c) allows us to probe the Pt d-band directly. It is known that the intensity of the Pt L3-edge white line is proportional to the density of unoccupied final states (Pt $5 \mathrm{~d}$ in this case) and scales with increasing vacancies in the Pt d-band [24]. We would expect cationic Pt species to exhibit more intense white lines. A large decrease in d-band vacancy upon reduction of the 1 ML-EQ deposit is seen. We see further decreases in d-band vacancy with further monolayer growth, trending towards characteristic spectra for Pt foil. Both the as-grown 2ML-EQ and reduced samples still exhibit increased d-band vacancies relative to the Pt foil. This is to be expected as previous work has suggested that the Pt d-band is susceptible to both strain effects and ligand effects $[23,25]$. This agrees with previous studies which predicted that the first monolayer of $\mathrm{Pt}$ on $\mathrm{Au}$ would exhibit greater d-band distortion compared to the following monolayers [5]. 
Fig. 2 a Pt 4f XPS spectra of reference materials and series of SLRR-grown samples on Au substrates. Vertical bands correspond to the location of zerovalent $\mathrm{Pt}$, which shifts for monolayer-scale samples. Note that reference spectra are not shown to scale with SLRR spectra. b Graphical representation of DFTcalculated surface charges. c Binding energy of the metallic component of the spectrum for a variety of sample preparation methods. Horizontal lines are added at 70.85 and $70.97 \mathrm{eV}$ to indicate regions where multiple samples show similar binding energies
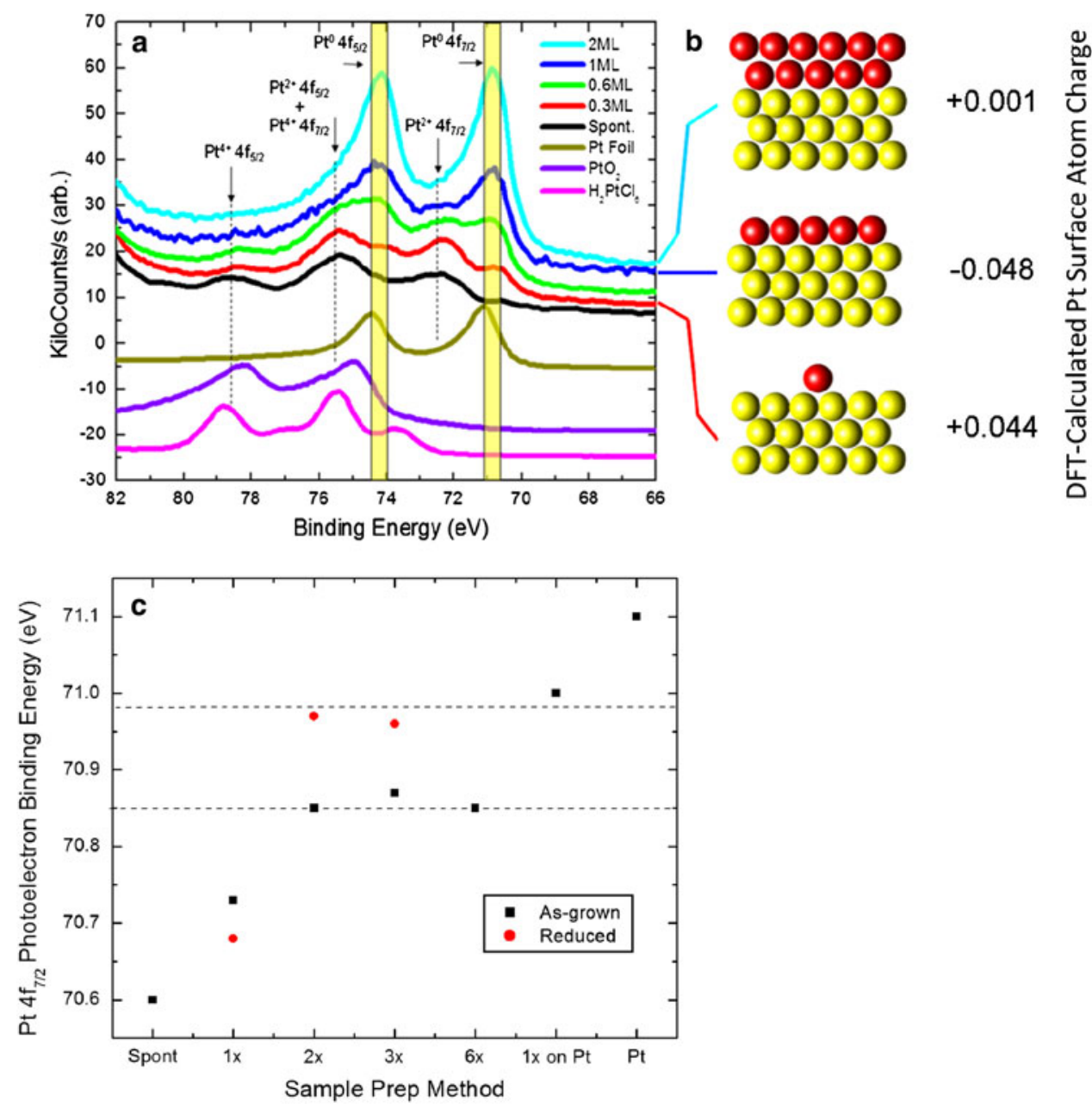

\section{Discussion 2: Film Reactivity and Durability}

\section{1 $\mathrm{CV}$ in $\mathrm{H}_{2} \mathrm{SO}_{4}$ : $\mathrm{OH}^{-}$Stripping}

Cyclic voltammetry performed in $1 \mathrm{M} \mathrm{H}_{2} \mathrm{SO}_{4}$ is shown in Fig. 4. This further supports the proposed SLRR reaction mechanism from previous section. An initial reductive sweep is plotted for the 1ML-EQ case, indicating reduction of surface-confined cationic Pt at $0.24 \mathrm{~V}$ (Arrow 1). This reduction occurs initially and is not repeated during further oxidation cycling, indicating an initial cationic component that is not reproduced. The following cycles allowed analysis of $\mathrm{Pt}$ behavior after initial reduction of these intermediaries. Reduction of submonolayer Pt (0.3ML-EQ) Pt sites (Arrow 2) occurs at $0.35 \mathrm{~V}$ and shifts positively to $0.52 \mathrm{~V}$ for thicker deposits (Arrow 3). In comparison, reduction of hydroxides from bulk-type $\mathrm{Pt}$ occurs for $\mathrm{Pt}$ wire at $0.56 \mathrm{~V}$ (Arrow 4).

Figure 4 illustrates an effect that we have previously reported [26] but now explore in more detail; the shift of the reduction feature to more negative voltages for ultrathin Pt deposits. It has been shown that the reduction peak after oxidation to $1.5 \mathrm{~V}$ corresponds to $\mathrm{OH}^{-}$desorption from the surface [27-29]. We demonstrate this feature for bulk Pt and varying coverages of Pt SLRR on a Au surface. For the 1 iteration case, we see a $-160 \mathrm{mV}$ deviation in the applied voltage required to strip adsorbed $\mathrm{OH}^{-}$from the surface compared to bulk Pt. This means that an additional $160 \mathrm{mV}$ must be applied to the 0.3ML-EQ electrode compared to the bulk Pt electrode to remove the $\mathrm{OH}^{-}$ adsorbate. For the 1ML-EQ and greater cases, this deviation decreases, but remains.

This shift indicates that for up to six iterations of growth, $\mathrm{OH}^{-}$requires more energy to remove from the SLRR surface than from a bulk Pt surface. Furthermore, the 0.3ML-EQ deposit showed evidence of a Pt species with an even greater $\mathrm{Pt}-\mathrm{OH}^{-}$bond strength. These two desorption potentials correspond to distinct Pt surface species: one that is thinly coated as a submonolayer across the surface and requires added potential to strip $\mathrm{OH}^{-}$, and a second species that has agglomerated to a sufficient thickness that the $\mathrm{OH}^{-}$affinity is reduced somewhat. Even at this point, a small additional voltage required to remove $\mathrm{OH}^{-}$is still present. It is likely that the species with greater 

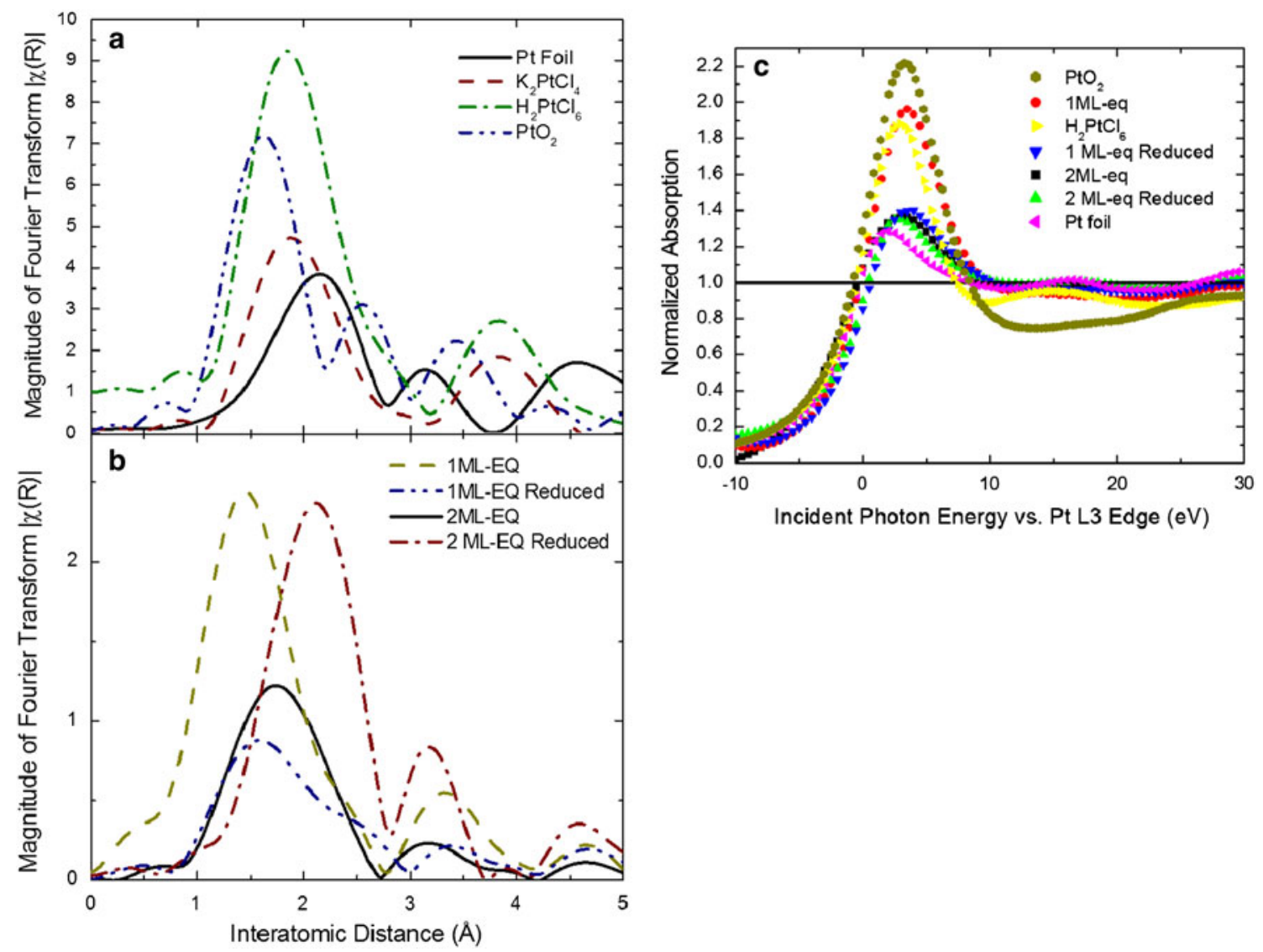

Fig. 3 a Normalized Fourier transformed EXAFS plots for reference samples in real space. b EXAFS for SLRR-fabricated samples with and without electrochemical reduction. c XANES spectra for the SLRR and reference samples

affinity for surface $\mathrm{OH}^{-}$is the same species which displays negative binding energy shifts in XPS. The following sections show that these two different species exhibit deviations from bulk Pt in their performance and durability.

\subsection{Durability During Repeated Electro-Oxidation of Methanol}

The deviations in electronic and structural properties of the Pt film have a profound influence on its durability. In order to simulate fuel cell conditions with high surface area electrodes, Pt monolayers were grown on carbon-supported $\mathrm{Au}$ grown by electrodeposition as described in previous work [2]. Following the Pt modification, accelerated aging tests by which the samples were cycled through a range of potentials were conducted.

Figure 5 plots the peak currents at $0.7 \mathrm{~V}$, for 0.3MLEQ, 1ML-EQ, and 2ML-EQ Pt on carbon-supported gold as a function of repeated methanol oxidation cycling. Higher electro-oxidation currents were achieved for the 2ML-EQ deposit. The 0.3ML-EQ and 1ML-EQ deposits decayed to a baseline current more quickly than the $2 \mathrm{ML}$ EQ deposit. Furthermore, the final baseline peak current after 200 cycles remains much higher for 2ML-EQ than for the 1ML-EQ or 0.3ML-EQ samples. We correlate the faster removal in 0.3ML-EQ and 1ML-EQ with unstable Pt. This Pt species was seen in XPS as deviated from metallic $(-0.4 \mathrm{eV})$ and in oxidation/reduction cycles as a species with increased affinity for $\mathrm{OH}^{-}$groups. The high rate of surface area loss for small deposits is explained by the presence of this deviated Pt. It is also apparent that the small amount of bulk Pt present in the 1ML-EQ and 2MLEQ samples corrodes more slowly.

\subsection{Film Interaction with Electroadsorbed CO}

The final tests involved electrochemical adsorption followed by either C-edge XANES spectroscopy in a vacuum environment (Fig. 6a) or anodic stripping of $\mathrm{CO}$ molecules from solution phase (Fig. 6b). Changes in metal-molecule interaction can be seen in the XANES results presented in Fig. 6a, with more intense peaks (corresponding to transition of electrons to the $2 \tilde{\pi}^{*}$ metal-molecule bonding orbital) indicating less substrate-CO electron backdonation. We see that submonolayer $\mathrm{Pt}$ deposits on $\mathrm{Au}$ (0.3ML-EQ Pt@Au) exhibit the lowest levels of backdonation from Pt. 


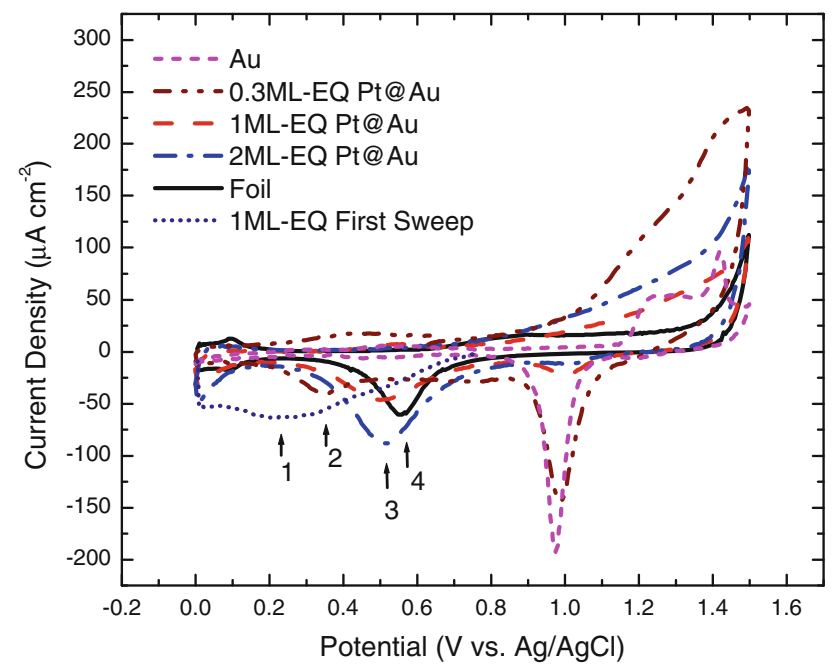

Fig. 4 Cyclic voltammetry in $1 \mathrm{M} \mathrm{H}_{2} \mathrm{SO}_{4}$ of $\mathrm{Au}$, $\mathrm{Pt}$, and samples with between 0.3ML-EQ and 2ML-EQ of Pt. Also included is a representative initial reductive sweep, starting at open circuit potential, to illustrate transient reduction of intermediary cationic $\mathrm{Pt}$ species (Arrow 1). Reduction of oxidic species at Au sites occurs near $1.0 \mathrm{~V}$ and can be seen to decrease with increasing Pt coverage. Reduction of Pt oxides at submonolayer (0.3ML-EQ) Pt sites (Arrow 2) occurs at $0.35 \mathrm{~V}$ and shifts positively to $0.52 \mathrm{~V}$ for thicker deposits (Arrow 3). Reduction of hydroxides from bulk-type $\mathrm{Pt}$ occurs for $\mathrm{Pt}$ wire at $0.56 \mathrm{~V}$ (Arrow 4)

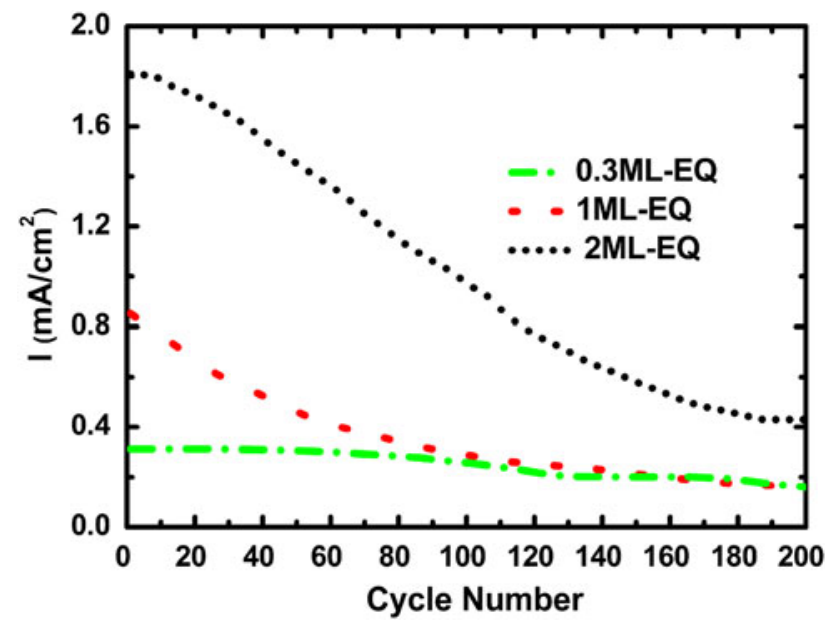

Fig. 5 Normalized peak current for electro-oxidation on $\mathrm{Pt}-\mathrm{Au}$ modified carbon fiber paper of $1 \mathrm{M} \mathrm{H}_{2} \mathrm{SO}_{4}+0.5 \mathrm{M}$ Methanol as a function of cycle number

This is due to the high cationic content of such deposits. For the case of 0.3ML-EQ Pt@Pt, we see significantly more backdonation than the same loading on a Au surface (consistent with increased metallic nature of $\mathrm{Pt}$ as seen in XPS). The 3ML-EQ Pt@Au deposit exhibits the most backdonation of any sample studied, indicating enhanced backdonation due to both metallic nature and architectureinduced electron transfer. Since increased backdonation leads to stronger $\mathrm{Pt}-\mathrm{CO}$ bond strengths, we expect, in a
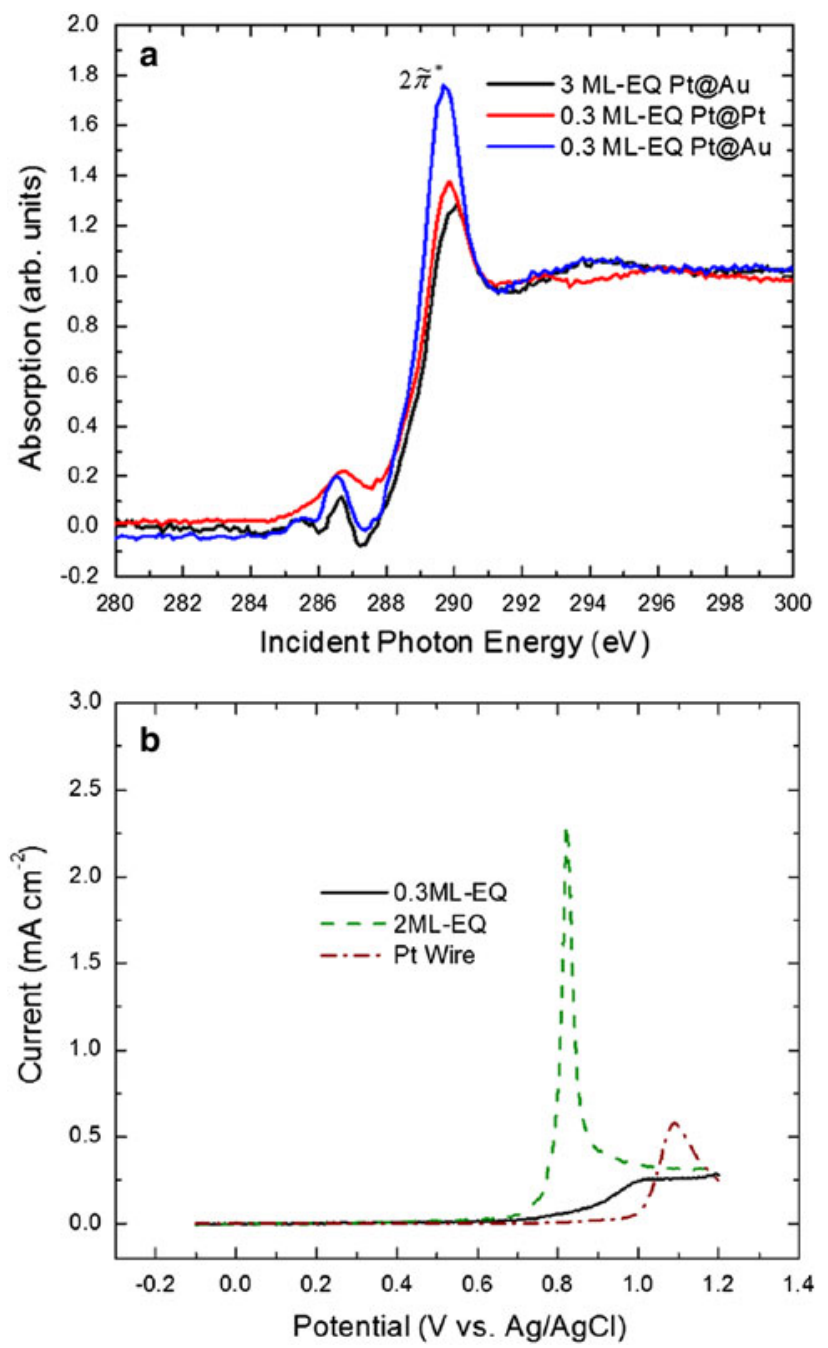

Fig. 6 a XANES of carbon monoxide $2 \pi^{*}$ orbital for adlayer Pt on $\mathrm{Au}$ and Pt substrates. b First-cycle $\mathrm{CV}$ of carbon monoxide electrooxidation on multiple electrodes in CO-saturated $1 \mathrm{M} \mathrm{H}_{2} \mathrm{SO}_{4}$ solution after adsorption of $\mathrm{CO}$ at $-0.1 \mathrm{~V}$

vacuum environment, the 3ML-EQ case to exhibit the strongest metal-adsorbate bond strengths. This is consistent with results by Pedersen et al. [5] which showed that the initial adlayers of $\mathrm{Pt}$ on $\mathrm{Au}$ exhibit stronger $\mathrm{Pt}-\mathrm{CO}$ bonds than bulk Pt. However, the subsequent anodic stripping study was not conducted in a vacuum environment. Thus, it is important to consider the influence of other species, most notably $\mathrm{OH}^{-}$, on the surface-adsorbate interaction.

Figure $6 \mathrm{~b}$ indicates a significant decrease in the potential at which CO electro-oxidation occurs for SLRR samples compared to the bulk Pt reference. For the 2ML-EQ case, the potential at which the CO electro-oxidation peak occurs is significantly lower than that for the bulk $\mathrm{Pt}$ or 0.3ML-EQ cases. In the case of submonolayer Pt coverages, we may attribute this lack of performance to the presence of cationic species, but in the case of bulk Pt, this 
does not apply. It is important to note that $\mathrm{CO}$ was introduced to the solution prior to the immersion of any electrodes. This accounts for differences between these results and prior work using SLRR-grown samples for $\mathrm{CO}$ anodic stripping (see also supplemental) [30].

It is possible to explain this observation by considering the role of $\mathrm{OH}^{-}$species adsorbed on the electrode surface. $\mathrm{OH}^{-}$adsorption on the Pt surface has been shown to be necessary for the electro-oxidation reaction to proceed quickly [31, 32]. The SLRR Pt films have been shown in this work to contain a disordered zerovalent $\mathrm{Pt}$ species with an increased affinity for $\mathrm{OH}^{-}$groups, which enable this lower-potential electro-oxidation reaction to occur. There are two competing effects at work when determining the $\mathrm{CO}$ electro-oxidation potential: the affinity for $\mathrm{OH}^{-}$ groups, and also the strength of the initial $\mathrm{Pt}-\mathrm{CO}$ bond. Even though the Pt layers studied here are expected to show an increased Pt-CO bond strength based on XANES analysis, the ability to nucleate an adsorbed $\mathrm{OH}^{-}$species through the $\mathrm{CO}$ adlayer leads to lower potentials required for electro-oxidation. Thus it has been shown that electrochemical affinities such as these can in fact overcome the bond strengths predicted by vacuum-based techniques.

In summary, core-electron spectroscopy and cyclic voltammetry combined with corroborating DFT calculations indicate that submonolayer, single monolayer, bi- and tri-layer Pt architectures on Au display unique properties arising from low-dimensionality and electron transfer phenomena. Solute derived submonolayer Pt has a strong preference for remaining cationic rather than neutral on a Au surface and consequently exhibit deviated catalytic activity compared to bulk Pt. This study has revealed that the nature of ultrathin Pt films undergoes a phase transition below a threshold distance of 2-3 monolayers from a $\mathrm{Au}$ surface, in terms of its atomic/electronic structure and the resulting chemical properties. In the range of 2-3 monolayers, $\mathrm{Pt}$ films are spared the rapid metal dissolution from repeated methanol oxidation cycles, but are thin enough to exhibit enhanced $\mathrm{CO}$ stripping due to electron transfer from the underlying Au. We demonstrate a combined methodology of solute derived monolayer-scale deposition, element-specific atomic/electronic structure measurements, and DFT calculations that will be effective in the inquiry of other multicomponent surface-driven materials systems.

Acknowledgments Use of the National Synchrotron Light Source, Brookhaven National Laboratory, was supported by the U.S. Department of Energy, Office of Science, Office of Basic Energy Sciences, under Contract No. DE-AC02-98CH10886. FA would like to acknowledge the ACS Petroleum Research Fund \#50934-DNI10 for financial support and the Synchrotron Catalysis Consortium for travel support. TM and DSS acknowledge support from the Center for Atomic Level Catalyst Design, an Energy Frontier Research Center funded by the U.S. Department of Energy, Office of Science, Office of
Basic Energy Sciences under Award Number DE-SC0001058 (Center for Atomic Level Catalyst Design).

Open Access This article is distributed under the terms of the Creative Commons Attribution License which permits any use, distribution, and reproduction in any medium, provided the original author(s) and the source are credited.

\section{References}

1. Stamenkovic VR et al (2007) Trends in electrocatalysis on extended and nanoscale Pt-bimetallic alloy surfaces. Nat Mater 6:241-247

2. Cheng S, Rettew RE, Sauerbrey M, Alamgir F (2011) Architecture-dependent surface chemistry for Pt monolayers on carbonsupported Au. ACS Appl Mater Interfaces 3:3948-3956

3. Rettew RE, Allam NK, Alamgir FM (2011) Interface architecture determined electrocatalytic activity of $\mathrm{Pt}$ on vertically oriented TiO2 nanotubes. ACS Appl Mater Interfaces 3:147-151

4. Janik MJ, Taylor CD, Neurock M (2007) First principles analysis of the electrocatalytic oxidation of methanol and carbon monoxide. Top Catal 46:306-319

5. Pedersen MØ et al (1999) How a gold substrate can increase the reactivity of a Pt overlayer. Surf Sci 426:395-409

6. Chen JG, Menning CA, Zellner MB (2008) Monolayer bimetallic surfaces: experimental and theoretical studies of trends in electronic and chemical properties. Surf Sci Rep 63:201-254

7. Adzic RR et al (2007) Platinum monolayer fuel cell electrocatalysts. Top Catal 46:249-262

8. Mrozek MF, Xie Y, Weaver MJ (2001) Surface-enhanced raman scattering on uniform platinum-group overlayers: preparation by redox replacement of underpotential-deposited metals on gold. Anal Chem 73:5953-5960

9. Gebregziabiher DK et al (2009) Electrochemical atomic layer deposition of copper nanofilms on ruthenium. J Cryst Growth 8:1271-1276

10. Thambidurai C, Kim YG, Stickney JL (2008) Electrodeposition of $\mathrm{Ru}$ by atomic layer deposition (ALD). Electrochim Acta 53:6157-6164

11. Hachiya T, Honbo H, Itaya K (1991) Detailed underpotential deposition of copper on gold(111) in aqueous solutions. J Electroanal Chem 315:275-291

12. Hafner $\mathbf{J}$ (2008) Ab-initio simulations of materials using VASP: density-functional theory and beyond. J Comput Chem 29: 2044-2078

13. Kresse G, Furthmuller J (1996) Efficient iterative schemes for ab initio total-energy calculations using a plane-wave basis set. Phys Rev B 54:11169-11186

14. Kresse G, Joubert D (1999) From ultrasoft pseudopotentials to the projector augmented-wave method. Phys Rev B 59:1758-1775

15. Vosko SH, Wilk L, Nusair M (1980) Accurate spin-dependent electron liquid correlation energies for local spin-Density calculations-a critical analysis. Can J Phys 58:1200-1211

16. Perdew JP et al (1992) Atoms, molecules, solids, and surfacesapplications of the generalized gradient approximation for exchange and correlation. Phys Rev B 46:6671-6687

17. Manz TA, Sholl DS (2010) Chemically meaningful atomic charges that reproduce the electrostatic potential in periodic and nonperiodic materials. J Chem Theory Comput 6:2455-2468

18. Gokcen D, Bae SE, Brankovic SR (2010) Stoichiometry of Pt submonolayer deposition via surface-limited redox replacement reaction. J Electrochem Soc 157:D582-D587 
19. Mohanty U (2011) Electrodeposition: a versatile and inexpensive tool for the synthesis of nanoparticles, nanorods, nanowires, and nanoclusters of metals. J Appl Electrochem 41:257-270

20. Bancroft GM et al (1975) ESCA study of sputtered platinum films. Anal Chem 47:586-588

21. Guczi L (2005) Bimetallic nano-particles: featuring structure and reactivity. Catal Today 101:53-64

22. Borgna A, Garetto TF, Apesteguía CR, Le Normand F, Moraweck B (1999) Sintering of chlorinated Pt/[gamma]-Al2O3 catalysts: an in situ study by X-ray absorption spectroscopy. J Catal 186:433-441

23. Hammer B, Morikawa Y, Norskov JK (1996) CO chemisorption at metal surfaces and overlayers. Phys Rev Lett 76:2141

24. Mukerjee S, Srinivasan S, Soriaga MP, McBreen J (1995) Role of structural and electronic-properties of Pt and Pt alloys on electrocatalysis of oxygen reduction- and in situ XANES and EXAFS investigation. J Electrochem Soc 142:1409-1422

25. Kitchin JR, Norskov JK, Barteau MA, Chen JG (2004) Role of strain and ligand effects in the modification of the electronic and chemical properties of bimetallic surfaces. Phys Rev Lett 93:156801

26. Rettew RE, Guthrie JW, Alamgir FM (2009) Layer-by-layer Pt growth on polycrystalline Au: surface-limited redox replacement of overpotentially deposited Ni monolayers. J Electrochem Soc 156:D513-D516

27. Burke LD, Buckley DT (1994) Anomalous stability of acidgrown hydrous platinum oxide films in aqueous media. J Electroanal Chem 366:239-251

28. Kaderovek M, Paseka I (1989) Electrochemical and XPS studies of the properties of oxide layers formed on a platinum electrode by anodic polarization in pure $\mathrm{H} 2 \mathrm{SO} 4$ and in $(\mathrm{H} 2 \mathrm{SO} 4+\mathrm{CNS}$. Electrochim Acta 34:121-126

29. Peuckert M, Coenen FP, Bonzel HP (1984) XPS study of the electrochemical surface oxidation of Platinum in $\mathrm{N} \mathrm{H} 2 \mathrm{SO} 4$ acid electrolyte. Electrochim Acta 29:1305-1314

30. Rincon A, Perez MC, Gutierrez C (2010) Dependence of lowpotential $\mathrm{CO}$ electrooxidation on the number of Pt monolayers on gold. Electrochim Acta 55:3152-3156

31. Koper MTM, Jansen APJ, van Santen RA, Lukkien JJ, Hilbers PAJ (1998) Monte Carlo simulations of a simple model for the electrocatalytic $\mathrm{CO}$ oxidation on platinum. J Chem Phys 109:6051-6062

32. Markovic NM, Grgur BN, Lucas CA, Ross PN (1999) Electrooxidation of $\mathrm{CO}$ and $\mathrm{H}-2 / \mathrm{CO}$ mixtures on $\mathrm{Pt}(111)$ in acid solutions. J Phys Chem B 103:487-495 